\title{
SOME CLAUSE-RELATIONAL ASSOCIATED NOMIMALS IN TECHNICAL ENGLISH
}

\author{
M.P. Jordan
}

\section{Background and Aims}

The purpose of this paper is to introduce a special type of clauserelational nominal group (or noun phrase) in technical English. A nominal of this type exhibits three primary characteristics: it is semantically connected in some way with a clause or nominal group in a previous sentence and thus provides some measure of lexical coherence between the sentences; it provides an indication of logical connection between the previous part of the text and the sentence of which it is a part; and it forms an essential part of the grammar and semantics of the new clause. Some brief background work is first necessary.

The concept of one nominal group in one sentence being "associated" with a nominal group in another sentence has already been well established from earlier work by Christophersen (1939), Hawkins (1978) and Jordan (1978) and more recent work by Jordan (1981, 1982a, 1983a, 1983b). Briefly, once a nominal has been introduced into the text (e.g., a table) further nominals can be introduced later which are semantically or collocationally related to this "trigger" (e.g., the leg, its cost, the surface finish of the table). Emphasis has been on defining the associations that connect a definable part, feature or characteristic of the trigger, and there is discussion of superordinate, hyponymic, and co-hyponymic connections in both thematic and nonthematic position and in very complex combinations. Although there is some mention of associated logical connection in Jordan 1981, this needs to be more fully explored, and that is the aim of this present paper.

\section{Nominal, Clause and Sentence Re-entry}

The final chapter of Jordan 1984 explains how the methods used by technical writers to re-enter previous nominal groups back into the text can also be used to re-enter clauses, sentences and larger passages of text. In particular, associated re-entry is explained in which clauses and sentences are re-entered in associative form:

(1) During the past several years the CF has been shifting its interest towards the $A E$ spectrometer for the analysis of oil samples for wear-metal levels and subsequent establishment of component trends. The reason for this change included increased intralaboratory repeatability, decreased operator dependence, and a greater range of particle size analysis.

Engineering Digest, Feb. 83, p.39) 
Most of the preceding sentence deals with the CF shifting its interest, and all this is re-entered as this change embedded within the larger associated nominal The reasons for this change. As with all associated nominals, this one is used to avoid a rather obvious statement such as "There are reasons for this change, which include ..." That is, the writer chose instead to include this information within an associated nominal group to achieve the appropriate level of conciseness and emphasis. As with associated re-entry of nominal groups, the associated re-entry of clauses and sentences can be triggered (including some form of the part being re-entered), or it can be left untriggered as in Example 2.

Enough has been said, it is hoped, to show that there is an urgent need for a cheaper form of shear connector. At present, savings produced by careful design tend to be absorbed completely by connector costs. The answer may lie in the development of a suitable high strength adhesive or bonding agent. Another approach, currently being examined by the author, is "part-casing". Journal of the American Concrete Institute, Feb. 72, p.87)

A need is identified in the first sentence of this example, and two associates are included later as The answer (to this need) and Another approach (to meeting this need). As the parts being re-entered into these associated clause re-entries are clear, they are left untriggered.

In both of these examples a definable part of the text has been re-entered into the text in such a manner as to indicate an identified relationship with the part that follows. When the first part of the two-part relation is small, it can be included within a larger introductory nominal group dominated by the lexical signal of logical connection:

The reason Canada's high technology industries are lagging has little to do with lack of capital or weakness in marketing. (Engineering Times, 19 Jul. 82, p.1)

This example has the same sort of connection indicated in Examples 1 and 2. This can be seen by re-writing it as "Canada's high technology industries are lagging. The reason for this has nothing to do with lack of capital or weakness in marketing," where the previous sentence is re-entered by the substitute item this within the associated nominal The reason for this. Further discussion of this concept appears under "The Referent's Associated Nominal" in Jordan 1978.

The dominant words creating the relation between the clauses in these texts are examples of what winter (1977) refers to as "Vocabulary 
3" words, the predictive function of which he explains in detail. We are here examining other functions of this important set of words, and in particular concentrating on their use as the semantically dominant part of associated nominals re-entering the meanings of whole clauses and sentences into new clauses and sentences.

\section{Vocabulary 3 Items as Headings and in Questions}

Common headings in many technical reports are simply Purpose, Results, Conclusions, etc. These are all untriggered associated nominals re-entering a portion of the document of which they are a part. The heading Purpose (or Aims or Goals) implicitly re-enters the whole document as part of the meaning of the nominal group, whereas Results usually re-enters the previous experimental work, and Conclusions (and Recommendations) usually implicitly re-enters the data obtained and the discussion under Results. When headings are used in this way, they are grammatically separate from the sentences surrounding them and are there only as a signpost of the type of information to follow:

\section{Results}

The solutions were investigated for the following ranges of parameters $L=1$ to $\sigma$ and $C=0$ to $\infty$. The results are summarised by the following graphs...

\section{Conclusions}

The elastic curves of a simply suspended rod, bent by its own weight, have been found. The application of Newton's method to this problem worked well up to the points where extreme sensitivity of the solution to the initial conditions were reached. Methods of overcoming this difficulty are being investigated and will be reported in future.

(Civil Engineering Transactions, 18(2) 76, p.101)

Note in this example how The results is an untriggered associated re-entry with of the investigation being implicit. In contrast Methods of achieving this difficulty is a triggered associated re-entry with this difficulty re-entering and characterizing the fact that Newton's method worked only to a point.

As Winter has pointed out $(1974,1983)$, the most overt method of communicating the relation between one clause or sentence and another is to use questions such as what is the Result? In practice this question is usually abbreviated simply as The Result?: 
(5)

Still too many sports enthusiasts strive for too much, much too soon. They're out there skating, skiing and running, emulating the superstars, all the while exposed to the added hazards of the winter season.

\section{The result?}

A dramatic increase in sports related injuries, many serious enough to require medical assistance.

(Canadian Family Physician, Jan. 83, p.5)

Although the question The result? is orthographically separated from the answer by the paragraphing of this advertisement, a measure of grammatical unity still occurs between the question and answer. In a more formal genre, this writing would have appeared as "The result (of this) is a dramatic increase ..." or "This has resulted in a dramatic increase..."

\section{A Cline of Markedness}

The cline (or spectrum) of markedness from the marked grammatical and orthographic separation of the Vocabulary 3 item from its "answer" to full grammatical unity is shown by Examples 4 and 5 and the following examples:

A good example is this fiber optic connector. By simplifying its design, we made it compatible with the latest cable designs, made its installation easier and reduced the total cost of making a fiber optic link by 88 percent.

The result? Now our customers can take advantage of the future with a connector that dramatically increases productivity. (Scientific American, Sep. 82, p.56)

(7) Once homeowner data are entered, the computer plugs in known data about the area's climate, soil, and heating-cooling loads on a seasonal basis. It also considers the pipe's thermal conductivity and the pumping losses normally encountered with various loop designs. The result: a price estimate that lets contractor and homeowner compare operating costs with conventional heating prices. (Popular Science, 0ct. 83, p.106)

(8) He decided he would make himself a tractor that would hold up for the long haul. While he was at it, he decided to build in some other features to solve common outdoor problems. Then, as the design developed, he realized that if the machine could solve his own yard problems, it would probably solve those of others as well. 
The result is the Apache Yard Truck, a beltless heavy-duty machine-- now in prototype--that offers great versatility and ruggedness.

(Popular Science, Oct. 83, p.46)

\section{Relational and Non-Relational Associations}

Example 8 illustrates the full integration of the Vocabulary 3 item into the grammar of its sentence. The nominal The result is an untriggered association which re-entries the previous three sentences as the input to the binary pair "Cause - Result" the second part of which is the complement of the clause of which The result is subject. A triggered example follows:

(9) One in every five households was also asked to provide information on income, occupation, education, ethnic origin, housing costs, immigration and labour force activity.

Data collected from the census provides Canada with the broadest, most complete set of information on the nation available, its population and its housing. The results of the census are used regularly by decision-makers in both public and private industry. (Engineering Digest, Jan. 84, p.9)

This example contains two non-relational triggered associations Data collected from the census and The results of the census. These nominals do not communicate a recognized relationship between the re-entered part (the census) and the predicates of the two sentences. Instead they establish a nominal that is associated with the trigger for the purpose of saying more about that association. Other examples cited earlier are "relational" associations, in which the purpose of the nominal is to indicate the relationship between the two parts being semantically connected. The following example, this time dominated by the Vocabulary 3 item reason, clarifies the principle involved:

(10) Take a magnet with you to the showroom if you care whether bumpers and hoods are aluminum or steel on the Chevrolet Cavalier, one of General Motor's new J-cars for 1981-82.

The Chevy version uses HSLA steel bumpers front and rear on most all models. The exceptions are those hatchback 77 models equipped with automatic transmissions and without air conditioning, or with manual transmissions with air conditioning. The rest of the hatchback line sports an all-plastic energy-management bumper design. 
The reason for this hodge-podge, according to Chevrolet, is that certain transmission/air-conditioning combinations add too much weight to the vehicle.

(Materials Engineering, Apr. 81, p.14)

The variations in bumper material selection are characterized as this hodgepodge embedded within the triggered associated nominal The reason for this hodge-podge. This larger nominal is grammatically connected as the matrix clause The reason ... that dominating the main clause certain ... vehicle. The purposes of the Targer nominal [this is a triggered relational nominal] are to provide the evaluation hodge-podge and to indicate the semantic relation of Basis between the part being reentered and the main clause.

\section{Other Logical Relations}

It is not the purpose of this paper to attempt to define the complex relations of logical connection in technical English (a task which badly needs to be done), but a brief discussion will indicate the general applicability of the principles discussed here to other relations and perhaps point the way for more detailed study. The logical relations can probably be explained initially by three reciprocal pairs, which are given below together with typical vocabulary 3 items and definitions in terms of questions:

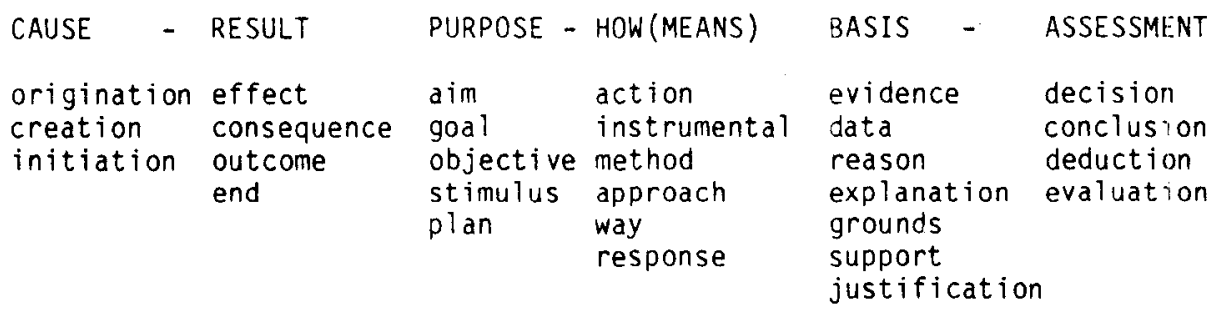

CAUSE: What caused this to happen?

RESULT: What was the result of this?

PURPOSE: What was the purpose of this?

HOW(MEANS): How was this achieved?

What was done to achieve this?

BASIS: What was this based on?

ASSESSMENT: What conclusion/decision was reached?
Inanimate cause-effect relation

Deliberate planning and achievement

Considered thought process 
Other closely connected relations which need to be considered in the final analysis of these semantic notions are those of achievement/ fulfillment discussed by Sweet (1891) and Jordan (1978); of justification and solutionhood discussed by Mann and Thompson (1983); and of modal semantics, emotive result and cognition discussed by Jordan (1978). Some work integrating some of these concepts is to appear shortly (Jordan 1985). A brief introduction to this topic follows. The reciprocal to the "Result" relation concentrated on so far is the relation of "Cause," in which the result is given first. Here is an example including the Vocabulary 3 item cause in an untriggered relational associated nominal mid-way on the cline of markedness:

(11) When Dr. Harold Miles, a physician in Naples, Fla., moved his stereo to change turntables, he found a blackened four inch area around the electrical outlet. The cause: lots of potentially damaging voltage arcing around the plug.

(Popular Science, Jul. 83, p.11).

Here is a complex example using goal and means for the reciprocal relations of Purpose-How(Means):

(12) With space missions dwindling and budgets tight, project managers want to boost space-vehicle efficiency by using the forces of nature. The goal: to use planetary or lunar atmospheres to steer and slow spacecraft by means of "aeroassist" designs.

(Popular Science, Oct. 83, p.28)

This example includes two Purpose-How(Means) relations, and it also contains a Basis-Assessment relation signalled by the subordinator with. The overall goal, indicated by want, is to boost space-vehicle efficiency, which is a decision based on the information given in the subordinate clause. The means by which they hope to achieve this goal is by using the forces of nature, the by ... ing clearly signalling the How(Means) relation as discussed by Winter (1977). The purpose of using the forces of nature is to use atmospheres (with a minor purpose relation being to change spacecraft velocity), and this in turn is to be achieved by "aeroassist" decisions--this time signalled by the Vocabulary 3 item means. The structure of these two sentences is shown below, with the items goal and means forming part of the signalling system of relations: 


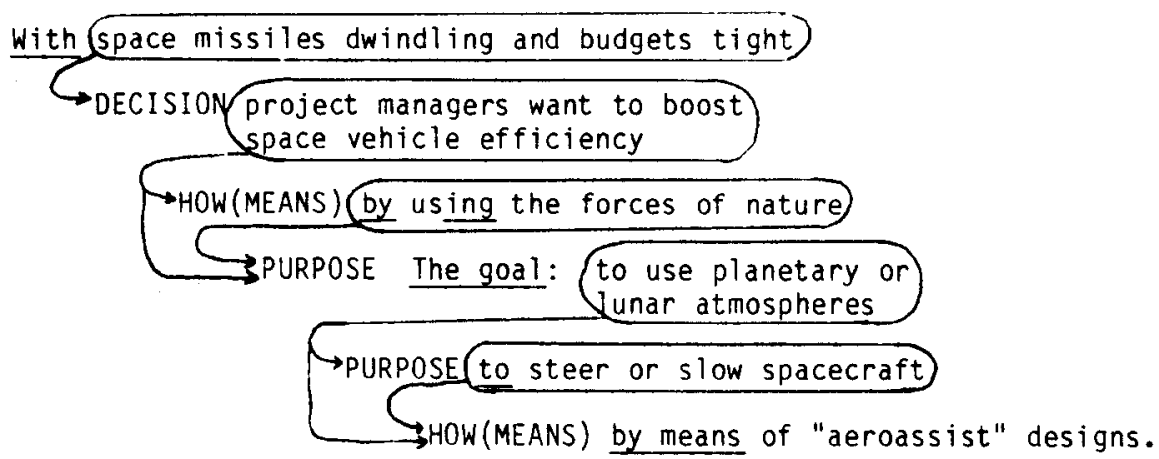

Embedded Substitute Predicates

Many relational associated nominals can be untriggered or can include triggers in the form of repetition, substitution, synonymy, generic nouns, etc. The relation of How(Means), however, is of ten found in the form of a substitute predicate embedded at the triggering within the relational nominal. Here is an example with two How(Means) relations communicated by complex nominals each containing an embedded way

With regard to the actual plant itself one good way of ensuring that an expanding plant maintains its flexibility is to deliberately plan the expansion as a feature of the original plant layout. The most efficient way to do this is to make the original plant a stage of the projected plant. (Ontario Engineering Digest, Nov/Dec 77, p.41)

The initial objective is signalled by ensuring and one means by which this can be accomplished is indicated by one good way ... is to surrounding this. The planning is not only a means of achieving the required maintenance of flexibility, but is also an objective itself, and the means of achieving that is given in the second sentence. The complex clause-relating nominal The most efficient way to do this is is subject of the sentence and contains the infinitive substitute clause to do this as complement of the Vocabulary 3 item way, and as clause-reentering substitute trigger for the nominal. Both How(Means) nominals in this example contain assessments of good and most efficient, and each indicates that other means are possible by one and The most. As with nominals triggered by substitute items or substitute nominals, nominals triggered by substitute clauses or predicates can either be relational or non-relational. Here is an example containing one of each type: 
(14) It is therefore necessary to keep the radioactive wastes from the fuel cycle sequestered for at least that period. One way of doing this is to incorporate them in glass. The French, for instance, have a full-scale plant doing this. (Engineering Journal, Jan./Feb. 77, p.8)

The need is signalled by necessary, and the How(Means) relation is indicated by the nominal-subject of the second sentence containing the Vocabulary 3 item way and the substitute predicate doing this. The third sentence contains a nonrelational associated nominal a full-scale plant doing this as object, with the substitute complement doing this re-entering the previous to-infinitive clause indicating one way of achieving the initial objective. The item how is indicative of a relation of How(Means) or How(Manner), the difference being the basis of such intended humour as "How did you climb out of the bathroom window?" - "Very carefully". Here is an example of a relational associated nominal of How(Means) dominated by how:

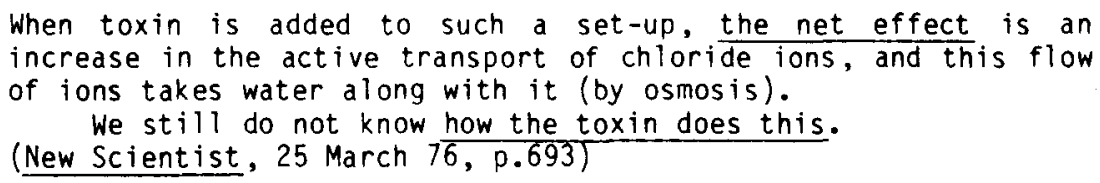

The relation of Result is indicated by the item effect within the untriggered clause-relational associated nominal The net effect, which has an implicit trigger of adding toxin to such a set-up. A further Result relation then follows with the synonym nominal this flow of ions as the subject-cause of the taking of water (with a How(Means) relation inside the parentheses). The substitute clause the toxin does this re-enters the meaning "the added toxin increases the active transport of chloride ions" into the clause-relational nominal how the toxin does this as abject of the second sentence. Here is the structure.

When toxic is added to such a set-up

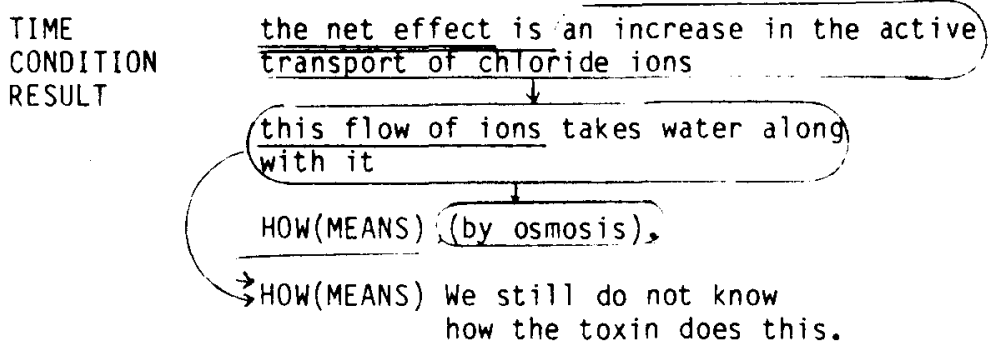


Note that the final sentence answers the question "How does the toxin do this?" with "We still do not know."

\section{Perspective}

Although this brief analysis can be seen as an extension of recent work into the use of associated nominals in technical texts, it is concerned much more with relations between clauses and sentences than with lexical connection, which has been the area of concentration for previous related work. The principles of clause relations mentioned here need further detailed work, and this analys is can perhaps indicate areas of fruitful study. More importantly, perhaps, it indicates how a study of associated nominals in context can be seen as a bridging area of analysis between lexical continuity, relational propositions, and the grammar of the nominal group and clause.

Although the work described here can be, and is being, included in technical writing instruction, it forms part of a wider body of theoretical knowledge now available to teachers of technical writing and should not be taught in isolation from it. As in all disciplines, isolated elements of knowledge can be quite misleading to linguistically immature students if they are not explained within a wider context of understanding. In the past, some teachers of our subject have been content with the established generalities of effective writing, and have chosen to concentrate on how best to teach these generalities rather than on developing the scholarly paradigm of technical writing. This paper is presented to those teachers of technical writing in Canada who have progressed past this "how to" stage of personal and professional development, and who are now seeking greater knowledge of technical English to enrich their understanding and teaching of the subject.

\section{REFERENCES}

Christophersen, P., The Articles: A Study of Their Theory and Use in English, Copenhagen, 1939

Hawkins, J.A., Definiteness and Indefiniteness, Croom-Helm, 1978

Jordan, M.P., The Principal Semantics of the Nominal This and That in Contemporary English Writing, PhD Thesis, CNAA/Hatfield/ Birmingham, 1978, University Microfilms 78-70,031

Jordan, M.P., Some Associated Nominals in Technical Writing, Journal of Technical Writing and Communication, Vol 11(3), 1981 
Jordan, M.P., Non-Thematic Re-entry, 9th International Systemic Conference, Toronto, Canada, 1982 (also in Systemic Perspectives in Discourse, (W.S. Greaves and J.D. Benson Eds.), Ablex 1984

Jordan, M.P., Co-Associative Cohesion in English Texts, Applied Linguistics Research Working Group Spring Colloquium, York University, Toronto, Canada, 1983 (also in Technostyle 2(2), 1983)

Jordan, M.P., Complex Lexical Cohesion in the English Clause and Sentence, LACUS Forum, 1983

Jordan, M.P., Fundamentals of Technical Description, Krieger, 1984

Jordan, M.P., Fundamentals of Technical Prose, in preparation, Krieger, 1985

Mann, W.C. and Thompson, S.A., Relational Propositions in Discourse, Instructional Sciences Institute, University of Southern California, Report ISI/RR-83-115, November 1983

Winter, E.0., Replacement as a Function of Repetition, PhD Thesis, University of London, England, Ref 1295, University Microfilms $77-70,036,1974$

Winter, E.0., A Study of Some Predictive Lexical Items in Written Discourse, Instructional Science 6(1), 1977, Special Issue

Winter, E.0., Towards a Contextual Gramar of English, George Allen and Unwin, 1982

Michael $P$. Jordan is Associate Professor of Linguistics and Engineering Communication in the Faculty of Applied Science at Queen's University, Kingston. He presented an earlier version of this paper at the Spring Colloquium of the Applied Linguistics Research Working Group, York University, Toronto, 1984. 